\section{BMJ Open} Ophthalmology

\title{
Dispersive viscosurgical devices demonstrate greater efficacy in protecting corneal endothelium in vitro
}

\author{
Timur Mert Yildirim, Gerd U Auffarth, Hyeck-Soo Son, Ramin Khoramnia, \\ Donald John Munro, Patrick R Merz
}

To cite: Yildirim TM,

Auffarth GU, Son H-S, et al. Dispersive viscosurgical devices demonstrate greater efficacy in protecting corneal endothelium in vitro. BMJ Open Ophthalmology 2019;4:e000227. doi:10.1136/ bmjophth-2018-000227

Received 20 September 2018 Revised 10 November 2018 Accepted 22 December 2018

\section{Check for updates}

C Author(s) (or their employer(s)) 2019. Re-use permitted under CC BY-NC. No commercial re-use. See rights and permissions. Published by BMJ.

The David J Apple International Laboratory for Ocular Pathology, Heidelberg University Eye Clinic, Heidelberg, Germany

Correspondence to Prof. Gerd U Auffarth; gerd. auffarth@med.uni-heidelberg.de

\section{ABSTRACT}

Objective During phacoemulsification, the corneal endothelium is protected by an ophthalmic viscosurgical device (OVD). In this in vitro study, we assessed six different OVDs for their effectiveness in protecting the corneal endothelium.

Methods and analysis Phacoemulsification was performed in cadaver eyes of young pigs. Five syringe units of six different OVDs were tested (Healon EndoCoat, Viscoat, Methylvisc, Healon, Healon GV, ProVisc). After surgery, the area of endothelium coated with OVD was determined in relation to the total endothelial surface. Additionally, an endothelial cell count was obtained. As a control, an endothelial cell count was obtained from freshly trephined corneas. Statistical analysis was performed using the Mann-Whitney U test and the Spearman correlation.

Results The least postoperative endothelial coating and cell count were observed in the cohesive OVDs while the dispersive OVDs showed statistically significant higher values. Healon EndoCoat and Viscoat yielded a coating area of $86(85-92) \%$ and $85(85-90) \%$, respectively. Endothelial cell count was highest in the two dispersive groups with 4065 (3928-4088) cells/ $/ \mathrm{mm}^{2}$ (Methylvisc) and 4032 (4015-4115) cells $/ \mathrm{mm}^{2}$ (Viscoat). Endothelial coating area and endothelial cell count correlated statistically significantly.

Conclusion Dispersive OVDs from this study showed greater adherence to the endothelial surface than the cohesive ones. Furthermore, postoperative endothelial cell counts of corneas treated with dispersive OVDs were higher than of corneas treated with cohesive OVDs. Our in vitro results suggest that dispersive OVDs protect the corneal endothelium better during phacoemulsification than cohesive OVDs.

\section{INTRODUCTION}

Ultrasound energy used during phacoemulsification is known to damage the corneal endothelium and other intraocular structures. $^{1}$ Ophthalmic viscosurgical devices (OVD) enlarge and maintain the anterior chamber in cataract surgery and protect anatomical structures, thus reducing this damage. $^{2}$ As the corneal endothelium only has limited capacity to regenerate, special care needs to be taken to protect it. ${ }^{3}$ OVDs

\section{Key messages}

What is already known about this subject?

- The use of ophthalmic viscosurgical devices (OVD) can reduce the damage to the corneal endothelium during cataract surgery.

\section{What are the new findings?}

This in vitro study suggests the superiority of dispersive OVDs in protecting the corneal endothelium during phacoemulsification compared with cohesive ones.

\section{How might these results change the focus of} research or clinical practice?

- In clinical practice, cataract surgeons ought to reconsider which OVD will best protect the corneal endothelium.

usually do not have chemical or pharmacological effects but act mechanically to provide volume to the anterior chamber and a barrier between tissues and surgical instruments. ${ }^{2}$ The protection of corneal endothelium can be explained by different mechanisms: Coverage of the endothelium protects it from air bubbles and free radicals that develop during phacoemulsification. ${ }^{4}$ The material properties of an OVD can absorb the kinetic ultrasound energy. ${ }^{5}$ The OVD creates increased distance between the phaco tip and the endothelium and other sensitive intraocular structures, thus weakening the energy acting on the tissue. ${ }^{6}$ Additionally, the tissue covered by OVD is protected from direct damage due to the touch of surgical instruments. ${ }^{1}$

Depending on the material properties, OVDs are generally classified as dispersive or cohesive viscoelastics. ${ }^{7}$ Dispersive OVDs allow better coating of intraocular anatomy due to their lower surface tension, cohesive ones have a higher potential to create space and can be removed more easily at the end of the surgery. ${ }^{49}$ Which of these substances better protect the endothelial cells during 


\begin{tabular}{|c|c|c|c|c|c|c|}
\hline Substance group & Dispersive & & & Cohesive & & \\
\hline Tradename & $\begin{array}{l}\text { Healon } \\
\text { EndoCoat }\end{array}$ & Viscoat & Methylvisc & Healon & $\begin{array}{l}\text { Healon } \\
\text { GV }\end{array}$ & ProVisc \\
\hline Manufacturer & $\begin{array}{l}\text { Johnson \& } \\
\text { Johnson }\end{array}$ & Alcon & Rayner & $\begin{array}{l}\text { Johnson \& } \\
\text { Johnson }\end{array}$ & $\begin{array}{l}\text { Johnson \& } \\
\text { Johnson }\end{array}$ & Alcon \\
\hline Ingredient(s) & $3 \% \mathrm{HA}$ & $3 \% \mathrm{HA}+4 \% \mathrm{CS}$ & $2 \%$ HPMC & $1 \% \mathrm{HA}$ & $1.4 \% \mathrm{HA}$ & $1 \% \mathrm{HA}$ \\
\hline Viscosity & $\begin{array}{l}50000 \\
\mathrm{mPa}\end{array}$ & $\begin{array}{l}60000 \\
\mathrm{mPa}\end{array}$ & $2600-7000 \mathrm{mPa}$ & $\begin{array}{l}300000 \\
\mathrm{mPa}\end{array}$ & $2500000 \mathrm{mPa}$ & $\begin{array}{l}100000 \\
\mathrm{mPa}\end{array}$ \\
\hline Molecular weight & $\begin{array}{l}800000 \\
\mathrm{Da}\end{array}$ & $\begin{array}{l}500000 \\
\mathrm{Da}\end{array}$ & NK & $\begin{array}{l}4000000 \\
\mathrm{Da}\end{array}$ & $\begin{array}{l}5000000 \\
\mathrm{Da}\end{array}$ & $\begin{array}{l}2400000 \\
\mathrm{Da}\end{array}$ \\
\hline
\end{tabular}

CS: chondroitin sulfate; Da, Dalton; HA: sodium hyaluronate; HPMC: hydroxypropyl methylcellulose; NK, not known; OVD, ophthalmic viscosurgical device; $\mathrm{mPa}$, millipascal.

standardised in vitro phacoemulsification has not been evaluated so far by a direct comparison between OVDs in current use. The aim of this study was to evaluate the protecting effect in porcine cadaver eyes of different OVDs in vitro in terms of their endothelial coating and the endothelial cell count after phacoemulsification.

\section{MATERIALS AND METHODS}

\section{Study materials}

Six OVDs were tested (table 1): Healon EndoCoat (Johnson \& Johnson Vision Surgical, Santa Ana, CA, USA); Viscoat (Alcon Laboratories, Fort Worth, TX, USA); Methylvisc (Rayner Intraocular Lenses, Worthing, West Sussex, UK); Healon (Johnson \& Johnson Vision Surgical); Healon GV (Johnson \& Johnson Vision Surgical); and ProVisc (Alcon Laboratories).

\section{Preparation of porcine eyes and surgical procedure}

Approval for using porcine cadaver eyes for scientific research purposes was granted in 2015 by the German State Office for Occupational Safety and Health, Berlin. The eyes were obtained from a commercial slaughterhouse (Schradi Frischfleisch, Mannheim, Germany). The age of the pigs at the time of slaughter was about 6 months. Immediately after enucleation, the eyes were stored in a humid chamber at $4^{\circ} \mathrm{C}$. Five syringe units of six different OVDs were stained with $10 \%$ fluorescein aqueous solution (Fluorescite, Novartis, Quebec, Canada) to a final concentration of $6 \mu \mathrm{g} / \mathrm{mL}$ and stored under dark conditions (table 1). In order to ensure a clear cornea, the eyes were stored in a moist environment until they were mounted on a holder just before the experiment. Only undamaged eyes with clear corneas and clear lenses were used for the experiment. A routine cataract surgeon performed the surgeries on the same day. A clear corneal incision was created and the anterior chamber was completely filled with prestained OVD. A continuous curvilinear capsulorhexis of $5 \mathrm{~mm}$ in diameter was performed using an Utrata Capsulorhexis Forceps (Geuder, Heidelberg, Germany). Then, phacoemulsification was performed using a Megatron S3 phaco machine (Geuder, Heidelberg, Germany). Standardised phaco machine settings for the procedure were as follows: aspiration rate $35 \mathrm{~mL} / \mathrm{min}$, vacuum+250 mm $\mathrm{Hg}$ and power $40 \%$, according to a previously published method. ${ }^{4}$ The standardised settings were not changed during surgery. No bimanual irrigation/aspiration (I/A) was performed after phacoemulsification in order to be able to only evaluate the effect of the phacoemulsification. After lens removal, the cornea was cut circularly and turned so that the entire endothelial surface could be observed. For each OVD five surgeries were performed.

\section{Endothelial OVD coverage}

A blue light filter (420-500 nm) was placed in front of the surgical microscope (Leica, Wetzlar, Germany) and photographs were obtained. Endothelial area coated with OVD in relation to the total area of the corneal endothelium was determined using an image analysis software (Image J, NIH, Bethesda, USA). Therefore, the total endothelial area of the inverted cornea was marked manually on each of the pictures using the image analysis software. The surface was calculated in pixels automatically. Subsequently, the area covered with stained OVD was marked manually and the surface was calculated in pixels automatically again. The percentage of coverage was afterwards calculated as relative value of both measurements.

\section{Endothelial cell count measurements}

After the picture was taken, the specimen was transferred to the cornea bank of our clinic and endothelial cell count was obtained. As a control, endothelial cell count was obtained from 10 freshly trephined porcine corneas without performing phacoemulsification. All corneas including the respective control corneas were stained with $0.5 \%$ alizarin red S (Carl Roth $\mathrm{GmbH}$, Karlsruhe, Germany) as well as $0.06 \%$ trypan blue (Gibco-Life Technologies, Thermo Fisher Scientific, Massachusetts, USA) for better visualisation of endothelial cells. ${ }^{10}$ The double staining with alizarin red and trypan blue allows determination between healthy and damaged cells of the entire corneal endothelium. Damaged cells show a blue-coloured cytoplasm and a deep blue-stained nucleus. Alizarin, on the other hand, stains the intercellular material and the cell membranes, thus providing a 
good red-blue contrast. ${ }^{10}$ For the staining procedure, two drops of $0.06 \%$ sterile isotonic trypan blue solution were applied to the endothelial surface. After $60 \mathrm{~s}$, the cornea was rinsed with balanced salt solution. Subsequently, two drops of a $0.5 \%$ sterile isotonic alizarin red $\mathrm{S}$ solution were added to the endothelial side. After another $60 \mathrm{~s}$, the dye was again rinsed with balanced salt solution.

Endothelial cell counting was performed manually under a light microscope (Olympus CKX41, Olympus Europa SE \& Co KG, Hamburg, Germany). We performed cell counting in real time with the support of the NAVIS software (Nidek Technologies, Padua, Italy). Endothelial cells were counted within a central rectangle. At the borders of the rectangle, only cells that were in contact with two adjacent cells were counted. The endothelial cell count of the vivid cells was calculated as endothelial cells $/ \mathrm{mm}^{2}$.

\section{Data analysis}

The coated endothelial area was calculated in per cent. Results are expressed as median (IQR, Q1-Q3). Statistical evaluation was performed using SPSS (IBM SPSS Statistics V.22) using the Mann-Whitney U test for non-parametric independent samples. Correlation between the two parameters was assessed using the Spearman's correlation for non-parametric data. A $\mathrm{p}$ value $<0.05$ was considered statistically significant.

\section{RESULTS}

Figures $1 \mathrm{~A}-\mathrm{C}$ and 2 show representative photographs, taken before image analysis, of the endothelial surface of corneas from each OVD group before software image analysis. The area covered with OVD after surgery in the dispersive and cohesive groups was respectively 85 $(65-88) \%$ and $20(12.5-30) \%$. Results of the endothelial coverage for each OVD are summarised in table 2.

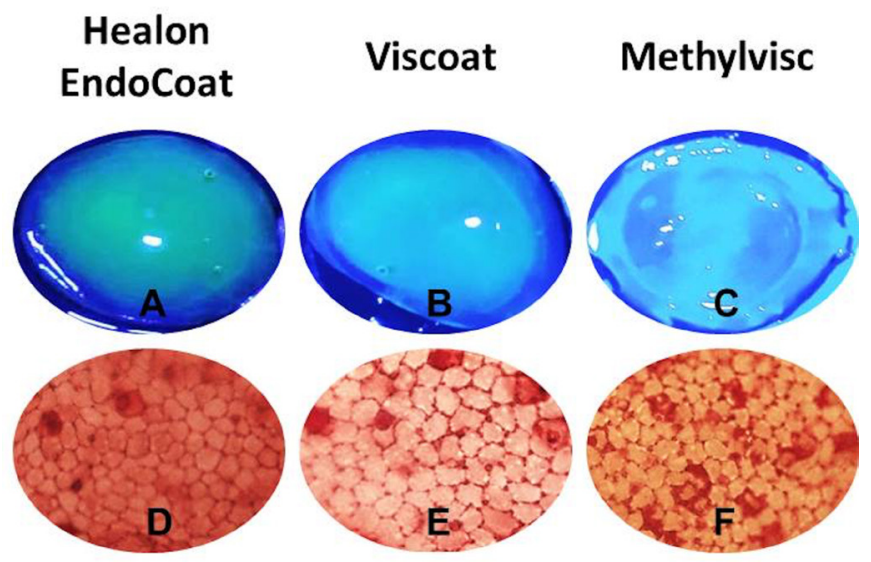

Figure 1 Photographs of corneas treated with dispersive ophthalmic viscosurgical devices (OVD). (A-C) Endothelial surface under a blue light filter with different amounts of OVD coating. (D-F) Corneal endothelium is visible as a single layer of hexagonal cells after staining with alizarin red $S$ and trypan blue (20-fold magnification). Damaged cells can be identified by the dark cytoplasm and nucleus.

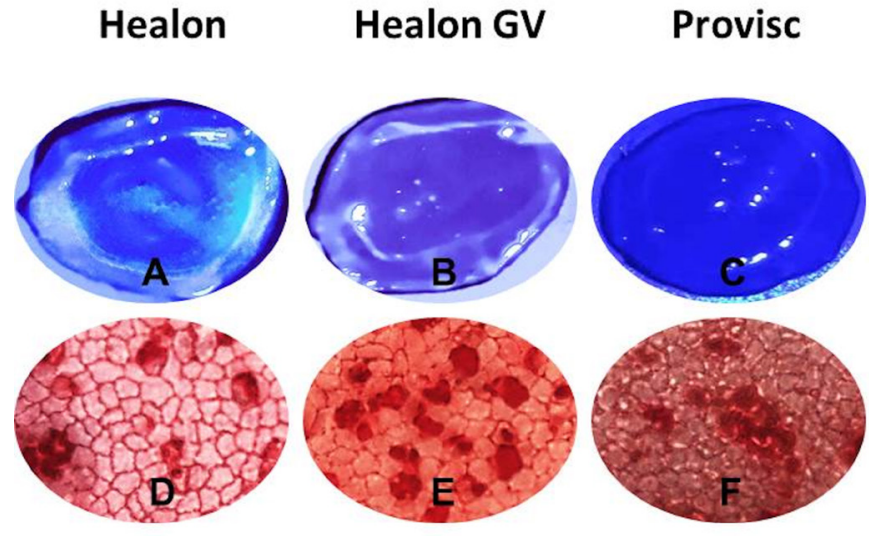

Figure 2 Photographs of corneas treated with cohesive ophthalmic viscosurgical devices (OVD). (A-C) Endothelial surface under a blue light filter with different amounts of OVD coating. (D-F) Corneal endothelium as a single layer of hexagonal cells after staining with alizarin red $S$ and trypan blue (20-fold magnification). Damaged cells can be identified by the dark cytoplasm and nucleus.

Healon EndoCoat had the highest value of 86 (85-92)\%, Healon GV the lowest one of 20 (10-20)\%.

In Figures 1D-F and 2 the corneal endothelium after histological staining can be seen as a single layer of cells (photographs taken at 20-fold magnification). Damaged cells can be identified by the dark cytoplasm and nucleus. All values of the endothelial cell count are presented in figure 3. The cell count was highest in the dispersive groups and control group. Healon EndoCoat and Viscoat showed similar endothelial cell count values of 4015 (3985-4198) cells $/ \mathrm{mm}^{2}$ and 4032 (4015-4115) cells/ $\mathrm{mm}^{2}$, respectively, whereas Methylvisc had a cell count of 4065 (3928-4088) cells $/ \mathrm{mm}^{2}$. Values for the cohesive viscoelastics were lower with 3898 (3866-3955) cells $/ \mathrm{mm}^{2}$ (ProVisc) followed by Healon GV with 3752 (3625-3855) cells $/ \mathrm{mm}^{2}$ and Healon with 3739 (3655-3900 cells $/ \mathrm{mm}^{2}$. There was a statistically significant lower mean endothelial cell count in the cohesive group compared with the control group.

The scatter plot (figure 4) shows the endothelial cell count related to the endothelial coverage of OVD. Endothelial coverage and endothelial cell count correlated significantly (correlation coefficient: 0.662). Looking at the correlation of the cohesive and dispersive groups separately, we found a statistically significant correlation with coefficients of 0.615 and 0.634 , respectively.

\section{DISCUSSION}

In this in vitro study, OVDs showed differences in corneal endothelial coating during phacoemulsification. Dispersive viscoelastics yielded better endothelial coating than the cohesive ones. As a clinically relevant parameter, we also assessed corneal endothelial cell count after surgery. Corneas from the cohesive OVD group showed significantly lower endothelial cell counts after surgery compared with untreated control corneas. Cell counts of corneas from the dispersive group were comparable with 
Table 2 Mean endothelial coverage

\begin{tabular}{llllll}
\hline Dispersive & & Cohesive & & P value \\
\hline $85 \%(65 \%-88 \%)$ & & & $20 \%(12.5 \%-30 \%)$ & $<0.05^{*}$ \\
$\begin{array}{l}\text { Healon } \\
\text { EndoCoat }\end{array}$ & Viscoat & Methylvisc & Healon & Healon & ProVisc \\
$\begin{array}{l}86 \% \\
(85 \%-92 \%)\end{array}$ & $85 \%$ & $50 \%$ & & GV & $20 \%$ \\
\end{tabular}

Median (Q1-Q3).

*Statistically significant difference, Mann-Whitney U test.

the ones of the control group and revealed no statistically significant difference.

Although porcine corneal endothelium resembles the human one, having similar cell density and shape, it is a limitation of our study that we used an animal model using porcine eyes. ${ }^{11}$ Our results cannot be directly transferred to human cases. The porcine lenses from this study were clear and easy to extract but in clinical practice, most lenses are older, densely opaque and require more phaco power to be removed, all leading to more potential damage to the corneal endothelium. Nevertheless, even with the lower phaco power used in this study, differences between the OVDs were seen. Methylvisc gave values in endothelial coating that were in between the dispersives (Healon EndoCoat or Viscoat) and the cohesives (Healon, Healon GV and ProVisc). Methylvisc's endothelial cell count was similar to that of Healon EndoCoat or Viscoat. Methylvisc was the only viscoelastic we studied that is made from hydroxypropyl methylcellulose
(HPMC). Different from hyaluronic acid (HA), HPMC is a synthetic derivative of cellulose and is not physiologically expressed in humans. Even though biocompatibility is considered to be high, there are reports of allergic reaction after cataract surgery due to the use of HPMC formulations. ${ }^{12}$ As HA is naturally expressed in the eye and there are receptors for HA on corneal endothelial cells, OVDs made from HA are known to offer excellent biocompatibility. ${ }^{13}$

Our results might lead to the conclusion that dispersive OVDs are generally better than cohesive ones in protecting the corneal endothelium. However, besides endothelial coating, other important parameters influence corneal health after cataract surgery such as the surgeon's skill and experience in removing the OVD completely towards the end of surgery and the effect that the extent of this removal might have on the duration of the surgery. Prolonged I/A and the general length of time of surgery can influence the success of the surgery.

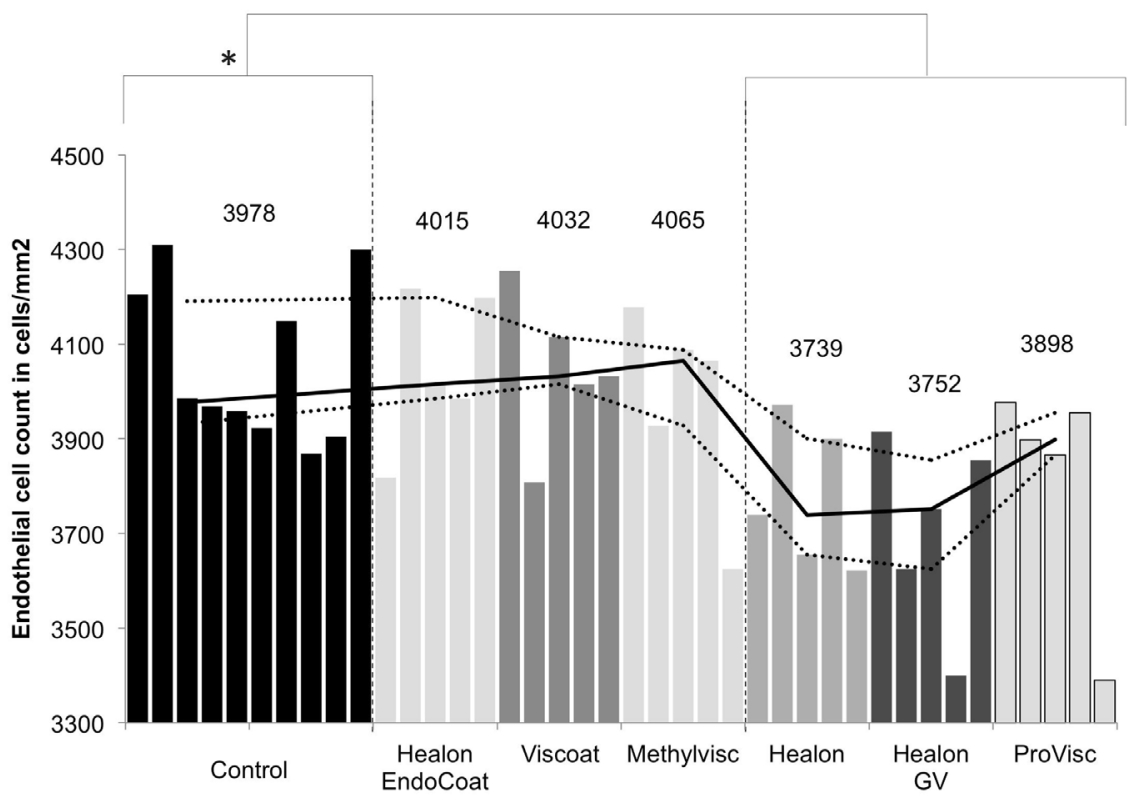

Figure 3 Postsurgery endothelial cell counts. The solid line shows the median of each group with first and third quartiles (surrounding dashed lines). *Indicates statistically significant difference between the control group and the cohesive group, Mann-Whitney U test. 


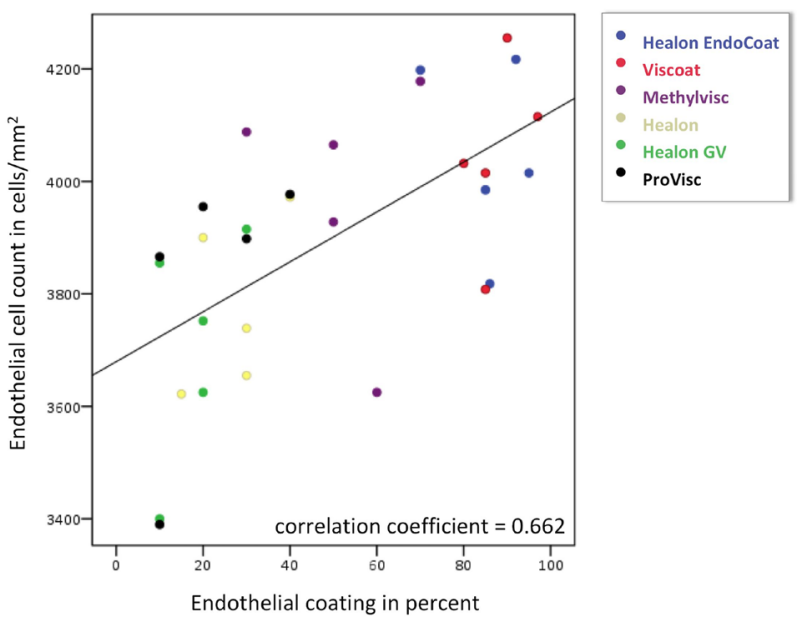

Figure 4 Scatter plot of the endothelial coating in relation to the endothelial cell count showing a correlation between the two parameters, Spearman's rank correlation $p<0.01$.

In a standardised laboratory procedure, conducted in 2004, removal time was shorter for cohesive than for dispersive viscoelastics: mean removal time after cataract surgery ranged from 18.3 to $46.5 \mathrm{~s}$ with a dispersive OVD (Viscoat) and from 15.3 to 25.6 with a cohesive one (ProVisc). ${ }^{9}{ }^{14}$ Removal time also depends on the interaction of the OVD with the intraocular lens (IOL) material. ${ }^{914}$ The time was found to be shorter in eyes with silicone or polymethyl methacrylate lenses compared with acrylic IOLs, especially with cohesive OVDs. ${ }^{9}{ }^{14}$

To combine these advantages of cohesive and dispersive OVDs, surgical techniques have been developed like the soft shell technique proposed by Arshinoff in $1999,{ }^{7}$ in which first a small amount of dispersive OVD is injected into the anterior chamber to coat the intraocular structures followed by a cohesive OVD that is used to create sufficient chamber for intraocular manipulation. Because the amount of dispersive OVD used in this technique is small, removal time remains low while endothelial coverage is high. ${ }^{4}$ Some manufacturers provide packages which combine one cohesive and one dispersive OVD. A recent clinical study compared the performance and safety of two OVD combinations: Duovisc (Alcon) and Twinvisc (Zeiss, Jena, Germany). Results showed similar performance and safety profiles in phacoemulsification cataract surgery for both groups. ${ }^{15}$ Regarding the corneal protection, the endothelial cell count was obtained before surgery and 3 months afterwards. Mean cell density loss from baseline was $11.7 \%$ and 9.6\% for Duovisc and Twinvisc, respectively. The authors concluded that the sequential use of one cohesive and one dispersive OVD using the soft shell technique leads to less endothelial cell loss and is a safe option for cataract surgery. ${ }^{1516}$ Results from the present laboratory study confirm that this corneal endothelial protection would be due to the good coating capability of the dispersive OVDs.
Another approach, using the beneficial properties of both OVDs best, is to create a mixture of one cohesive and one dispersive OVD with superior characteristics for cataract surgery. A recent study by Tognetto $e t$ al evaluated the in vitro properties of a mixture of Healon GV and Viscoat, called DisCoVisc (Alcon). The authors came to the conclusion that this combination has rheological properties that might be advantageous during cataract surgery. ${ }^{17}$

One prospective randomised clinical study compared the outcomes of cataract surgery using the soft shell technique with the outcomes of cataract surgery using one single injection of DisCoVisc. ${ }^{18}$ One hundred eyes underwent phacoemulsification by the same surgeon. Both techniques provided similar results regarding the endothelial cell protection. It ought to be mentioned that the intraocular pressure was not recorded in this study. Thus, the safety of the two techniques could not be compared.

The use of the combination of one cohesive and one dispersive OVD in one surgery seems to be beneficial but further clinical studies are needed to address the question which of the combined approaches is superior in terms of safety.

Even though corneal damage is decreasing as a result of these developments, there is always a certain amount of cell loss postoperatively. ${ }^{15}$ Improvements in OVD properties and surgical techniques can aim to minimise this cell loss.

In conclusion, the corneal endothelial coverage results and endothelial cell count data of our in vitro study show that dispersive viscoelastics offer good corneal protection during standardised phacoemulsification. Postoperative corneal endothelial cell count was higher in the dispersive groups. Especially Healon EndoCoat and Viscoat showed a high endothelial coverage and minimal postoperative cell loss.

Acknowledgements We thank Irina Vöhringer for her contributions to the data acquisition

Contributors GUA designed, planned and supervised the study and secured funding. TMY and HSS conducted the experiment and acquired data. PRM acquired materials for the study, supervised the experiment and acquired data. TMY, GUA and PRM statistically analysed and interpreted the data. DJM and RK interpreted the data. TMY drafted the manuscript. GUA, DJM and RK revised the manuscript. TMY submitted the manuscript. All authors critically revised the manuscript and approved its final version.

Funding Klaus Tschira Foundation, Heidelberg, Germany.

Disclaimer The funding organisation had no role in the design or conduct of this research.

Competing interests TMY reports non-financial support from Alcon, outside the submitted work. GUA and RK report grants, personal fees and non-financial support from Alcon, Rayner and Johnson \& Johnson, and grants and non-financial support from Carl Zeiss Meditec, outside the submitted work. HSS, DJM and PRM have nothing to disclose.

Patient consent for publication Not required.

Provenance and peer review Not commissioned; externally peer reviewed.

Open access This is an open access article distributed in accordance with the Creative Commons Attribution Non Commercial (CC BY-NC 4.0) license, which permits others to distribute, remix, adapt, build upon this work non-commercially, and license their derivative works on different terms, provided the original work is 
properly cited, appropriate credit is given, any changes made indicated, and the use is non-commercial. See: http://creativecommons.org/licenses/by-nc/4.0/.

\section{REFERENCES}

1. Beesley RD, Olson RJ, Brady SE. The effects of prolonged phacoemulsification time on the corneal endothelium. Ann Ophthalmol 1986;18:216-9.

2. Auffarth GU. Ophthalmic viscoelastic devices (OVD) in ocular surgery. Bremen Germany: UNI-MED-Verlag, 2012.

3. Choi J, Jeon H, Song J, et al. Biofunctionalized lysophosphatidic acid/silk fibroin film for cornea endothelial cell regeneration. Nanomaterials 2018;8:290.

4. Kretz FT, Limberger IJ, Auffarth GU. Corneal endothelial cell coating during phacoemulsification using a new dispersive hyaluronic acid ophthalmic viscosurgical device. J Cataract Refract Surg 2014;40:1879-84.

5. Glasser DB, Osborn DC, Nordeen JF, et al. Endothelial protection and viscoelastic retention during phacoemulsification and intraocular lens implantation. Arch Ophthalmol 1991;109:1438-40.

6. Eisner G. Principles of the practical use of viscoelastic substances in eye surgery. Fortschr Ophthalmol 1989;86:19-22.

7. Arshinoff SA. Dispersive-cohesive viscoelastic soft shell technique. $J$ Cataract Refract Surg 1999;25:167-73.

8. Pandey S, Thakur J, Werner L. Update on ophthalmic viscosurgical devices. In: Agarwal S, Agarwal A, Agarwal A, eds. Phacoemulsification. edn. Boca Raton FL: Taylor and Francis, 2004: 179-95.
9. Auffarth GU, Holzer MP, Visessook N, et al. Removal times for a dispersive and a cohesive ophthalmic viscosurgical device correlated with intraocular lens material. J Cataract Refract Surg 2004;30:2410-4.

10. Taylor MJ, Hunt CJ. Dual staining of corneal endothelium with trypan blue and alizarin red $\mathrm{S}$ : importance of $\mathrm{pH}$ for the dye-lake reaction. Br J Ophthalmol 1981;65:815-9.

11. Kim MK, Hara H. Current status of corneal xenotransplantation. Int $J$ Surg 2015;23:255-60.

12. Munk SJ, Heegaard S, Mosbech $\mathrm{H}$, et al. Two episodes of anaphylaxis following exposure to hydroxypropyl methylcellulose during cataract surgery. J Cataract Refract Surg 2013;39:948-51.

13. Forsberg N, Von Malmborg A, Madsen K, et al. Receptors for hyaluronan on corneal endothelial cells. Exp Eye Res 1994;59:689-96.

15. Auffarth GU, Auerbach FN, Rabsilber T, et al. Comparison of the performance and safety of 2 ophthalmic viscosurgical devices in cataract surgery. J Cataract Refract Surg 2017;43:87-94.

16. Labiris G, Sideroudi H, Rousopoulos $\mathrm{K}$, et al. Cohesive versus dispersive-cohesive ophthalmic viscosurgical device in torsional intelligent phaco. J Cataract Refract Surg 2015;41:681-2.

17. Tognetto D, Cecchini P, D'Aloisio R, et al. Mixed polymeric systems: new ophthalmic viscosurgical device created by mixing commercially available devices. J Cataract Refract Surg 2017;43:109-14.

18. Praveen MR, Koul A, Vasavada AR, et al. DisCoVisc versus the softshell technique using viscoat and provisc in phacoemulsification: randomized clinical trial. J Cataract Refract Surg 2008;34:1145-51. 